Article

\title{
Information Thermodynamics of the Cell Signal Transduction as a Szilard Engine
}

\author{
Tatsuaki Tsuruyama \\ Clinical Research Center for Medical Equipment Development, Department of Pathology, \\ Kyoto University Hospital, Shogoin-Kawahara-cho 54, Sakyo-ku, Kyoto 606-8057, Japan; \\ tsuruyam@kuhp.kyoto-u.ac.jp; Tel./Fax: +81-75-751-3111
}

Received: 27 February 2018; Accepted: 22 March 2018; Published: 26 March 2018

\begin{abstract}
A cell signaling system is in a non-equilibrium state, and it includes multistep biochemical signaling cascades (BSCs), which involve phosphorylation of signaling molecules, such as mitogen-activated protein kinase (MAPK) pathways. In this study, the author considered signal transduction description using information thermodynamic theory. The ideal BSCs can be considered one type of the Szilard engine, and the presumed feedback controller, Maxwell's demon, can extract the work during signal transduction. In this model, the mutual entropy and chemical potential of the signal molecules can be redefined by the extracted chemical work in a mechanicochemical model, Szilard engine, of BSC. In conclusion, signal transduction is computable using the information thermodynamic method.
\end{abstract}

Keywords: Szilard engine; mutual entropy; signal transduction; information thermodynamics; fluctuation theorem

\section{Introduction}

Biological systems exist in an open and stable non-equilibrium state, homeostasis, which fluctuates. These systems most likely utilize fluctuations for various cellular activities, molecular motors [1,2], and possibly for the signal transduction [3-6], i.e., the transmission of biological information. Biological signal transduction systems are characterized by the presence of biochemical signaling cascades (BSCs). These BSCs are interconnected, forming a network. For the evaluation of environmental stimuli and their consequences, different computational methodologies and the field of systems biology have been developed.

Signal transduction refers to the sequential biochemical reactions that include the modification or demodification of multi-enzymatic molecules. The enzymes may be the substrates in the subsequent reactions, and the modification sequence may be considered the signal flow sequence with the form change of different modified proteins [7]. The sequential response to the environmental stimuli is mediated by many intracellular metabolites, such as adenosine triphosphate (ATP), and its modified form, cyclic AMP, represents one of the second messengers [8].

The biological systems maintain homeostasis, steady isothermal and isovolumic states. The author considered the feedback systems, a unique property of the biological systems, allowing the maintenance of the steady state in the system. BSC can be considered the utilization of the tentative increase in fluctuation as the mediator of the signal transmission [7]. The feedback contributes to the decrease in the fluctuation. If the feedback system is operated by a feedback controller, termed Maxwell's demon, a biological signaling system can be simplified and presented as a model to obtain the definite computation of the signal transduction. According to the previously proposed information thermodynamic theory $[5,6]$, the upper limit of the average work $\langle w\rangle$ that can be extracted from the cyclic system by the feedback controller depends on the system temperature $T$, Boltzmann constant $k_{B}$, 
free energy change $\Delta F$, and the mutual information $H$, which is measured by the feedback controller, as shown in Inequality (1) [9]:

$$
\langle w\rangle \leq \Delta F+k_{B} T H .
$$

This inequality represents the generalized second law of thermodynamics $[2,10]$. In order to achieve equality in Inequality (1), the feedback process is quasi-static, and the usage of the acquired information from the Szilard engine should be non-wasteful [11]. In this study, the author considered the extra-work utilized for the signal transduction in the informational biological thermodynamic system, which fluctuates around a steady state, i.e., homeostasis. For the isothermal and isovolumic biological system, Inequality (1) can be simplified to:

$$
\langle w\rangle \leq k_{B} T H .
$$

The specific aim of this study was to develop a theoretical formulation of the chemical potential for signal transduction, based on the thermodynamic information theory, to be applied in the investigations of signal transduction.

\section{A Model Signaling Cascade}

If an isothermal and isovolumetric biological system is considered, a model of the BSC, consisting of $j$ and the reverse $-j$ steps can be constructed $(1 \leq j \leq n)$.

$$
\begin{aligned}
& X_{1}(R)+L \rightarrow X_{1}-L *: 1^{s t} \\
& X_{1}-L * \rightarrow X_{1}+L:-1^{s t} \\
& X_{1}-L+X_{2}+A \rightarrow X_{1}-L+X_{2} *+D: 2^{\text {nd }} \\
& X_{2} *+P h_{2} \rightarrow X_{2}+P i \rightarrow-2^{\text {nd }} \\
& \cdots \\
& X_{j} *+X_{j+1}+A \rightarrow X_{j} *+X_{j+1} *+D: \rightarrow j^{\text {th }} \\
& X_{j+1} *+P h_{j} \rightarrow X_{j+1}+P i: \rightarrow-j^{\text {th }} \\
& \cdots \\
& X_{n-1} *+X_{n}+A \rightarrow X_{n-1} *+X_{n} *+D: \rightarrow n-1^{\text {th }} \\
& X_{n} *+P h_{n} \rightarrow X_{n}+D: \leftarrow-(n-1)^{\text {th }} \\
& X_{n} *+D N A+R N A p o l+N \text { ribonucleotide } \rightleftharpoons \\
& X_{n}+D N A *+R N A p o l: \rightarrow n^{\text {th }}, \leftarrow-n^{\text {th }}
\end{aligned}
$$

Each step represents an enzymatic modification/demodification of the signaling molecules in the cytoplasm, maintained by a chemical reservoir that provides signaling molecules, such as ATP (symbol $A$ in the Formula (3)). ATP is a well-known mediator of signal transduction, and it is hydrolyzed into adenosine diphosphate (ADP; $D$ in the Formula (3)) and an inorganic phosphate (Pi), which is utilized for the modification of the amino acid residue of $X_{j}$. Here, $X_{j}$ and $X_{j}^{*}$ denote a signaling molecule, either unmodified (inactive) or modified (active) by the signaling molecule, respectively. The first reaction $(j=1)$ in the BSC represents the uptake or binding of an extracellular molecule, the ligand (L), by $X_{1}$, which represents a receptor (R) located on the cellular membrane, which is involved in sensing of the external stimulation by L. Afterward, $X_{1}-\mathrm{L}$ promotes the modification of $X_{2}$ in the cytoplasm into $X_{2}{ }^{*}$, phosphorylated by Pi from ATP, and ADP is produced. The BSC processes continue in this manner, such that the $j^{\text {th }}$ signaling molecule, $X_{j}^{*}$, induces the modification of $X_{j+1}$ into $X_{j+1}{ }^{*}$. Following the final step, the signaling molecule $X_{n}{ }^{*}$ translocates to the cell nucleus, where it binds to the promoter region of DNA and induces gene transcription of mRNA from ribonucleotides in the final step in (3). During the BSC steps, demodification of $X_{j+1}$ into $X_{j+1}$ occurs through an enzymatic reaction mediated by a phosphatase $\left(P h_{j} ; 1 \leq j \leq n-1\right)$, in which $P i$ is released. A pre-stimulation steady state is recovered in this manner [7] (Figure 1). 


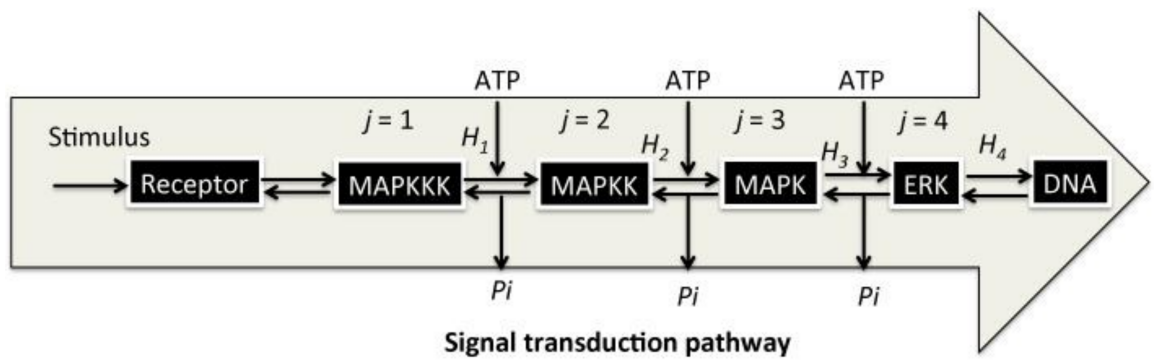

Figure 1. Schematic showing an example of signal transduction pathway. MAPKKK, Mitogen-activated Protein (MAP) kinase kinase kinase; MAPKK, MAP kinase kinase; MAPK, MAP kinase; ERK, extracellular signal-regulated kinase; DNA, deoxyribonucleic acid. The right pointing arrows represent the direction of BSC and the left pointing arrows represent the reverse direction. Adenosine triphosphate (ATP) represents the supplied adenosine triphosphate from the outside world and $\mathrm{Pi}$ represents the released inorganic phosphate from the pathway to the outside world. Stimulus represents binding of growth factor or other chemokines to the receptor. $H_{j}(j=1,2,3,4)$ represents the transmitted mutual information along the direction.

The occurrence probability $\left(p_{j}\right)$, which represents the selection probability of the $j^{\text {th }}$ step using the signaling molecule concentration, $X_{j}$ is defined respectively:

$$
p_{j}=X_{j} / X
$$

with

$$
p_{j} *=X_{j} * / X
$$

and

$$
\sum_{j=1}^{n}\left(p_{j}+p_{j} *\right)=1
$$

Here, the author introduces $X$ that represents the total concentration of the signaling molecules.

$$
X=\sum_{j=1}^{n} X_{j}+X_{j} *=\text { const }
$$

Because the sum of the concentrations is considered constant, protein production is relatively slower than the signal transduction step. Therefore,

$$
p_{j}+p_{j} *=p_{j}^{0}=\text { const. }
$$

\section{Signaling Cascade as a Szilard Engine}

Here, the author hypothesized that the feedback controller determines the activation or inactivation of signaling molecules in contact with an ATP chemical reservoir, with ATP freely transferred between the reservoir and the individual $j^{\text {th }}$ reaction field. The cell signaling system can be divided into an $n$ number of fields, corresponding to the individual $j^{\text {th }}$ step $(1 \leq j \leq n)$, for the formation of the BSC from the $1^{\text {st }}$ to $n^{\text {th }}$ step. In a biochemical system, signaling molecules are macromolecules localized in the cytoplasm, and, since their diffusion rate is sufficiently slow, the signaling reaction is considered a localized system as well. In the current model, each field contains all $X_{j+1}{ }^{*}$ and $X_{j+1}$ species $(1 \leq j \leq n-1)$, with the concentrations identical to those of $X_{j+1}{ }^{* s t}$ and $X_{j+1}{ }^{s t}$, respectively, at the initial state, when the stimuli are absent. The lowercase subscript $s t$ on the right of $X_{j+1}{ }^{* s t}$ and $X_{j+1}{ }^{s t}$ represents the steady-state concentration. In this system, the feedback controller has the potential to recognize the ratio of signaling molecule concentration differences between the initial and final states in the $j^{\text {th }}$ field. Subsequently, the controller provides feedback by selecting the transferring of $X_{j+1}{ }^{*}$ or $X_{j+1}$, to determine the orientation of the signal transduction. Each individual 
$j^{\text {th }}$ step in Formula (3), consists of a four-step chemical cycle (Figure 2), which can be summarized as follows:

(i) The feedback controller measures the changes in the concentration of the active signaling molecule $X_{j+1} *$ in the $j^{\text {th }}$ field.

(ii) If $X_{j+1}$ concentration increases from $X_{j+1}{ }^{\text {st* }}$ to $X_{j+1}{ }^{\text {st* }}+\Delta X_{j+1} *\left(\Delta X_{j+1} * 0\right)$, the $j^{\text {th }}(1 \leq j \leq n)$ step proceeds in the same signaling direction, while the feedback controller introduces $\Delta X_{j+1}{ }^{*}$ of $X_{j+1}{ }^{*}$ to the $(j+1)^{t h}$ field from the $j^{\text {th }}$ field by opening the forward gate in the $j^{\text {th }}$ field to the $(j+1)^{t h}$ field. In contrast, if $X_{j+1}$ concentration decreases from $X_{j+1}{ }^{\text {st* }}$ to $X_{j+1}{ }^{\text {st* }}-\Delta X_{j+1}{ }^{*}\left(\Delta X_{j+1}{ }^{*}>0\right)$, the $j^{t h}$ step proceeds in the opposite direction of BSC. In that case, the controller introduces $\Delta X_{j}$ of $X_{j}$ to the $(j+1)^{t h}$ field from the $j^{\text {th }}$ field by opening the back gate.

(iii) Subsequently, $X_{j+1}$ * flows back with the forward transfer of $X_{j+1}$ from the $(j+1)^{\text {th }}$ field to the $j^{\text {th }}$ field due to the mixing entropy gradient. This $X_{j+1}{ }^{*}$ can rotate the machinery that can extract chemical work equivalent to $w_{j}$. If $X_{j}$ flows back from the $(j+1)^{\text {th }}$ field to the $j^{\text {th }}$ field simultaneously with the transfer of $X_{j+1}$ * due to the mixing entropy gradient with the rotation of the molecular machinery, this molecule can extract the chemical work equivalent to $w_{-j}$.

(iv) In the $(j+1)^{t h}$ to the $(j+2)^{\text {th }}$ field, a similar reaction is initiated for signal transduction in an identical or the opposite orientation.

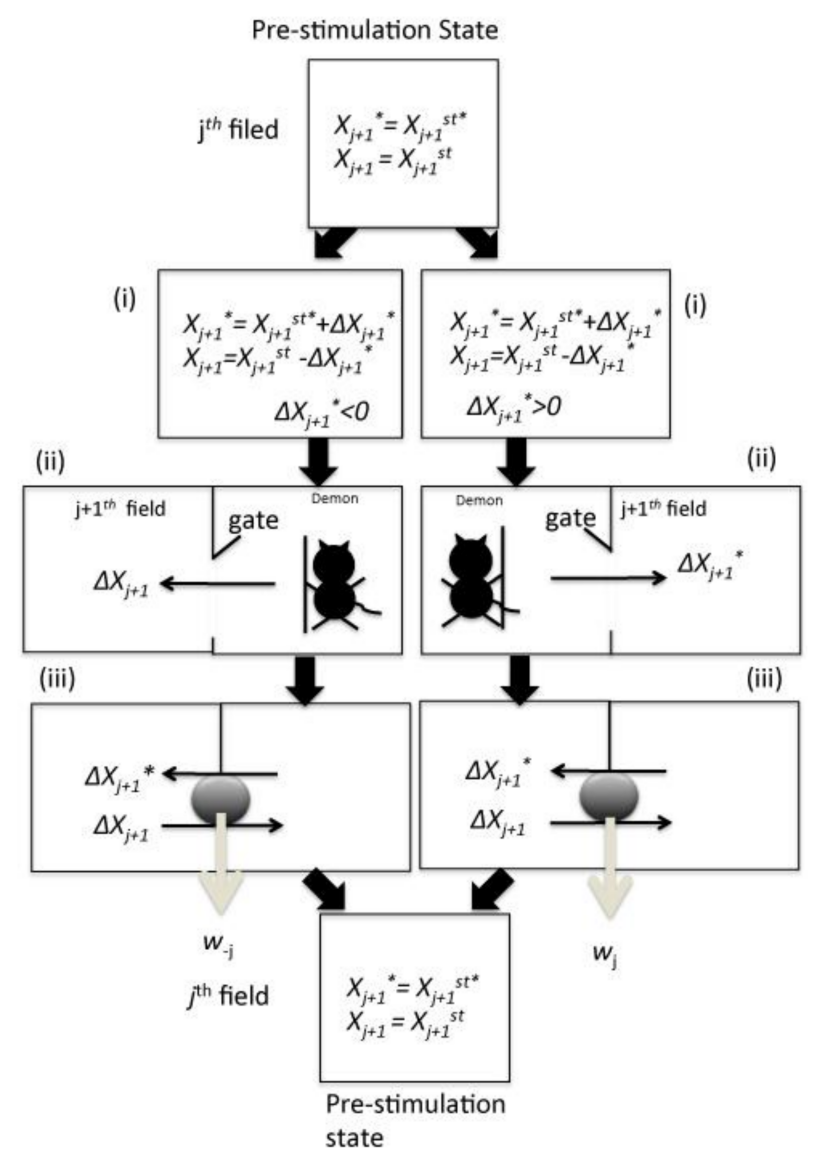

Figure 2. Schematic showing feedback controller processes. (i) The feedback controller observes the increase of $X_{j+1}{ }^{*}$. (ii) The controller opens the gate for the increased $\Delta X_{j+1}{ }^{*}$ or for the increased $\Delta X_{j+1}$ to enter the $(j+1)^{t h}$ field from the $j^{\text {th }}$ field, to prevent the signal from proceeding further. The $j^{\text {th }}$ field in the system recovers to the initial state in this reaction cycle. (iii) The chemical work $\left(w_{j}\right)$ can be extracted by the backflow of $\Delta X_{j+1}{ }^{*}$ of $X_{j+1}$ from $(j+1)^{\text {th }}$ field to the $j^{\text {th }}$ field. If the feedback controller observes the increase of $X_{j+1}$, the controller opens the back gate and the next steps follow and the chemical work $\left(w_{-j}\right)$ can be extracted by the backflow of $\Delta X_{j+1}$ of $X_{j+1}$ from the $(j+1)^{\text {th }}$ field to $j^{\text {th }}$ field. The gray globule on the barrier represents the machinery between the steps. 
During signaling transduction in a cell, the total concentration of signaling molecules is kept nearly constant $\left(X_{j+1}+X_{j+1}{ }^{*}=\right.$ const. $)$, and here, the author can use:

$$
d X_{j+1}+d X_{j+1} *=0
$$

\section{Mutual Entropy and Chemical Work by a Model of Szilard Engine}

We further investigated whether cell signal transduction can be modeled as a Szilard engine [11]. As a well-known example, let us consider a type of Szilard engine in which a single particle is enclosed in a field in contact with a heat bath. To obtain the information $H$, irrespective of its location in the left or right side of the field, a barrier was inserted in the middle of the field. The information $I$ can then be recorded by a feedback controller and utilized as a resource for the extraction of work through the isothermal expansion of the left or right side. This can be done by moving the barrier in order to recover the initial state. The movement orientation of the barrier is determined by the obtained information, which represents a feedback process.

Next, the author subsequently considered the amount of information $H_{j}$ that can be transmitted, using this model system of signal transduction. The cyclic reaction, including the exchange between $X_{j+1}$ and $X_{j+1}{ }^{*}$, satisfies the requirements of the Szilard engine, because the acquired data on the $X_{j+1}{ }^{*}$ change in (ii) is used as a resource for the quasi-static work in (iii). In general, the upper limit of the extracted average chemical work by the machinery between the $j^{\text {th }}$ and $j+1^{\text {th }}$ reaction field, $\left\langle w_{j}\right\rangle$, can be obtained based on the previous research on Helmholtz free energy $\Delta F_{j}$ and mutual information $H_{j}[2,10,12-16]$ :

$$
\left\langle w_{j}\right\rangle \leq-\Delta F_{j}+k_{B} T H_{j}
$$

In (10), the lowercase $j$ represents the number of the cyclic reaction step in the BSC. Therefore, the extracted average chemical work that can be extracted from a Szilard engine was given by (10):

$$
\left\langle w_{j}\right\rangle=k_{B} T H_{j} .
$$

The mutual entropy that is received by feedback controller is substantially equivalent to the difference mixing entropy between $I_{j}$ at the $j^{\text {th }}$ reaction field and $I_{j+1}$ at the $j+1^{\text {th }}$ reaction field. Considering the entropy current arising from the difference in the mixing entropy of $j^{\text {th }}$, to $(j+1)^{\text {th }}$ steps consisting of $X_{j+1}{ }^{*}$ and $X_{j+1}$ differences, the mixing entropy of activated ${ }^{\text {th }}$ step is described as follows [7]:

$$
I_{j}=-X\left[\left(p_{j+1}+\Delta p_{j+1}\right) \log \left(p_{j+1}+\Delta p_{j+1}\right)+\left(p_{j+1} *+\Delta p_{j+1} *\right) \log \left(p_{j+1} *+\Delta p_{j+1} *\right)\right]
$$

where $\Delta p_{j+1}{ }^{*}$ and $\Delta p_{j+1}$ denote the fluctuations of the occurrence probability. In the $(j+1)^{t h}$ step, in the absence of $X_{j}$ and $X_{j}^{*}$ fluctuations, the following calculation can be applied:

$$
I_{j+1}=-X\left[p_{j+1} \log p_{j+1}+p_{j+1} * \log p_{j+1} *\right]
$$

Afterward, the mutual entropy $H_{j}$ obtained from Equations (12) and (13) using differential coefficient of mixing entropy for $p_{j+1} *[7,17]$ :

$$
H_{j}=I_{j}-I_{j+1}=\frac{\partial I_{j}}{\partial p_{j+1} *} \Delta p_{j+1} * \approx X \log \frac{p_{j+1}}{p_{j+1} *} \Delta p_{j+1} *=\log \frac{p_{j+1}}{p_{j+1} *} \Delta X_{j+1} *
$$

Then, the chemical extracted average chemical work, $\left\langle w_{j}\right\rangle$, in (10) from (i) to (iv) in Section 3 was calculated using Equation (14) as follows:

$$
\left\langle w_{j}\right\rangle=\oint k_{B} T \log \frac{p_{j+1}}{p_{j+1^{*}}} d X_{j+1} *
$$


Here the author defined the expanded chemical potential of the $j^{\text {th }}$ signal molecules in similar manner around the equilibrium state using $\mu^{0}$ at standard condition:

$$
\begin{gathered}
\mu\left(X_{j+1}\right)=\mu^{0}\left(X_{j+1}\right)+k_{B} T \log p_{j+1} \\
\mu\left(X_{j+1} *\right)=\mu^{0}\left(X_{j+1} *\right)+k_{B} T \log p_{j+1} *
\end{gathered}
$$

Using the chemical potentials and the sufficient long signal duration of the $j^{\text {th }}$ step $\tau_{j}(\rightarrow \infty)$, the author has:

$$
\frac{1}{\tau_{j}} \log \frac{p_{j+1}}{p_{j+1}}=\frac{\mu\left(X_{j+1}\right)-\mu\left(X_{j+1} *\right)}{k_{B} T \tau_{j}} .
$$

Previously, the author obtained the following result using the average entropy production rate (AEPR) $\zeta_{j}$, the current density of entropy production rate, $c_{j+1}$ [7]:

$$
\zeta_{j}=\frac{c_{j+1} \Delta X_{j+1} *}{k_{B} T \tau_{j}} .
$$

Here, $c_{j+1}$ is given by chemical potential difference between species:

$$
c_{j+1}=\mu\left(X_{j+1}\right)-\mu\left(X_{j+1} *\right)
$$

From Equations (15), (18) and (20), the author obtained the following:

$$
\left\langle w_{j}\right\rangle=\oint c_{j+1} d X_{j+1} *=c_{j+1} \Delta X_{j+1} *
$$

The integral symbol signifies the integration of the work through the cycle step. Likewise, the extracted work by the machinery between the $j^{\text {th }}$ and $(j+1)^{\text {th }}$ reaction fields, $w_{-j}$, in the opposite orientation in (ii) is given by:

$$
\begin{gathered}
\left\langle w_{-j}\right\rangle=\oint k_{B} T \log \frac{p_{j+1} *}{p_{j+1}} d X_{j+1}=\oint k_{B} T \log \frac{p_{j+1}}{p_{j+1} *} d X_{j+1} * \\
\left\langle w_{-j}\right\rangle=-c_{j+1} \Delta X_{j+1}=c_{j+1} \Delta X_{j+1} *
\end{gathered}
$$

In this manner,

$$
\left\langle w_{j}\right\rangle=\left\langle w_{-j}\right\rangle,
$$

Then, from Equation (11),

$$
H_{j}=\frac{c_{j+1} \Delta X_{j+1} *}{k_{B} T}=\omega_{j+1} \Delta X_{j+1} *,
$$

with

$$
\omega_{j+1}=\frac{c_{j+1}}{k_{B} T}
$$

This $\omega_{j+1}$ represents the signal mobility [7]. Because the density of entropy signal current $c_{j+1}$ is equivalent to the diffusion coefficient $D_{j+1}$ according to our previous study [7], Equation (25) implies that the mutual entropy is carried by the signaling molecules:

$$
H_{j}=\frac{D_{j+1} \Delta X_{j+1} *}{k_{B} T}
$$

Thus, the mutual entropy can be related to the diffusion process of signaling molecule. 


\section{Conclusions}

Generally, cellular systems maintain homeostasis, in which fluctuations are minimized, while the extracellular stimuli induce the fluctuations in the system. Considering the feedback controller, signal transduction can be formulated according to the fluctuations in the concentration of signaling molecule, and the signal transduction level in the BSC may be evaluated quantitatively. In the mechanicochemical process, the difference between the chemical potentials of activated species and non-activated species is the source of the chemical work. The entire transfer step forms a cycle step. The entire average work $<w_{j}>$ done through the cycle by a given system is equal to the sum of the works by transferring the extensive amount $\Delta X_{j+1}{ }^{*}$ through its conjugate potential as follows $[18,19]$.

$$
\left\langle w_{j}\right\rangle=\oint_{C}\left[\mu\left(X_{j+1}\right)-\mu\left(X_{j+1} *\right)\right] d X_{j+1} *
$$

Equations (15) and (22) can be considered as a form of Equation (28). This work formula in Equations (15) and (22) is consistent with the Inequality $(1)[5,12,13]$. The chemical work may be associated with AEPR during the cycle reaction of the steps. By measuring the kinetic parameter based on Formula (3), it is possible to actually compute the average work $<w_{j}>$ by entropy production in hydrolysis of ATP during the signal transduction. Using the probability of step $j+1$, given step $j$, is defined as $p(j+1 \mid j)$ and probability of step $j$, given step $j+1$, is defined as $p(j+1 \mid j)$, FT yields the following, using AEPR $\zeta_{j}$ :

$$
\lim _{\tau_{j} \rightarrow \infty} \frac{1}{\tau_{j}} \log \frac{p(j+1 \mid j)}{p(j \mid j+1)}=-\zeta_{j}
$$

with

$$
\zeta_{j} \triangleq \frac{1}{\tau_{j}} \int_{0}^{\tau_{j}} \Delta \zeta_{j}\left(s_{j}\right) d s_{j}
$$

Here, $s_{j}$ is an arbitrary parameter representing the progression of a signal event. The transitional rate of step $j+1$, given step $j$, as $v(j+1 \mid j)$ and the transitional rate of step $j$, given step $j+1$, as $v$ $(j \mid j+1)$ are defined. When the cell system stays at the detailed balance in the homeostasis, as follows:

$$
p(j+1 \mid j) v(j+1 \mid j)=p(j \mid j+1) v(j \mid j+1) .
$$

Therefore, from Equations (29) and (31):

$$
\lim _{\tau_{j} \rightarrow \infty} \frac{1}{\tau_{j}} \log \frac{v(j+1 \mid j)}{v(j \mid j+1)} \simeq \zeta_{j}
$$

From Equations (19), (25) and (32), and the kinetic coefficient $k_{j}$ for $j^{\text {th }}$-step in Formula (3), following is obtained:

$$
\lim _{\tau_{j} \rightarrow \infty} \frac{1}{\tau_{j}} \log \frac{k_{j} A X_{j} * X_{j+1}}{k_{-j} P h_{j} X_{j+1} *}=\frac{c_{j+1} \Delta X_{j+1} *}{k_{B} T \tau_{j}}
$$

Then we have

$$
\log \frac{k_{j} A X_{j} * X_{j+1}}{k_{-j} P h_{j} X_{j+1} *} \simeq H_{j}
$$

Here, the author has the following from Equations (11) and (34):

$$
\left\langle w_{j}\right\rangle=k_{B} T \log \frac{k_{j} A X_{j} * X_{j+1}}{k_{-j} P h_{j} X_{j+1} *}
$$

By measuring the kinetic parameter based upon Equation (35), it is possible to actually compute the average work $\langle w\rangle$. This simple relation may be verified by using experimental data as follows. There have been reports that chemical potential difference can be converted to work under isothermal status [20]. With this recent knowledge, this study attempted to present a model of the signal 
transduction step as a Szilard engine that can extract work. This modeling is actually effective for quantifying chemical work as described in the text. However, it is necessary to verify this modeling by further experimental studies.

In conclusion, the work that can be extracted in the signal-transduction step can be obtained using Inequality (1). Recently, many theories for computing protein-protein networks and gene expression networks have been developed [21,22]. Signaling entropy was recently investigated from the viewpoint of genome informatics [23,24], and its availability was confirmed [25,26]. In these studies, signaling entropy was defined using the transient probability obtained from each node in a network graph of the transcriptome profile of a single cell in order to quantify the gene activation levels of its molecular pathways. In the current study, the author aimed to connect these computational methods using informational thermodynamics and the kinetics of actual chemical reactions. The presented BSC model can describe the relationship between the chemical potential, mutual entropy, and work information, which is based on the information thermodynamics.

Acknowledgments: This work was supported by a Grant-in-Aid from the Ministry of Education, Culture, Sports, Science, and Technology of Japan (Synergy of Fluctuation and Structure: Quest for Universal Laws in Non-Equilibrium Systems, P2013-201 Grant-in-Aid for Scientific Research on Innovative Areas, MEXT, Japan). The author thanks Prof. Kenichi Yoshikawa of Doshisha University and Prof. Masaki Sano of Tokyo University for their theoretical advices.

Conflicts of Interest: The author declares no conflict of interest.

\section{References}

1. Seifert, U. Stochastic thermodynamics of single enzymes and molecular motors. Eur. Phys. J. E Soft Matter 2011, 34, 1-11. [CrossRef] [PubMed]

2. Seifert, U. Stochastic thermodynamics, fluctuation theorems and molecular machines. Rep. Prog. Phys. 2012, 75, 126001. [CrossRef] [PubMed]

3. Tsuruyama, T. A model of cell biological signaling predicts a phase transition of signaling and provides mathematical formulae. PLoS ONE 2014, 9, e102911. [CrossRef] [PubMed]

4. Tsuruyama, T. Kinetic stability analysis of protein assembly on the center manifold around the critical point. BMC Syst. Biol. 2017, 11, 13. [CrossRef] [PubMed]

5. Ito, S.; Sagawa, T. Information Thermodynamics on Causal Networks. Phys. Rev. Lett. 2013, 111, 18063. [CrossRef] [PubMed]

6. Sagawa, T.; Kikuchi, Y.; Inoue, Y.; Takahashi, H.; Muraoka, T.; Kinbara, K.; Ishijima, A.; Fukuoka, H. Single-cell E. coli response to an instantaneously applied chemotactic signal. Biophys. J. 2014, 107, 730-739. [CrossRef] [PubMed]

7. Tsuruyama, T. Information Thermodynamics Derives the Entropy Current of Cell Signal Transduction as a Model of a Binary Coding System. Entropy 2018, 20, 145. [CrossRef]

8. Kato, K.; Omura, H.; Ishitani, R.; Nureki, O. Cyclic GMP-AMP as an Endogenous Second Messenger in Innate Immune Signaling by Cytosolic DNA. Annu. Rev. Biochem. 2017, 86, 541-566. [CrossRef] [PubMed]

9. Sagawa, T.; Ueda, M. Minimal energy cost for thermodynamic information processing: Measurement and information erasure. Phys. Rev. Lett. 2009, 102, 250602. [CrossRef] [PubMed]

10. Sagawa, T.; Ueda, M. Generalized Jarzynski equality under nonequilibrium feedback control. Phys. Rev. Lett. 2010, 104, 090602. [CrossRef] [PubMed]

11. Szilard, L. On the decrease of entropy in a thermodynamic system by the intervention of intelligent beings. Behav. Sci. 1964, 9, 301-310. [CrossRef] [PubMed]

12. Sagawa, T.; Ueda, M. Fluctuation theorem with information exchange: Role of correlations in stochastic thermodynamics. Phys. Rev. Lett. 2012, 109, 180602. [CrossRef] [PubMed]

13. Ito, S.; Sagawa, T. Maxwell's demon in biochemical signal transduction with feedback loop. Nat Commun. 2015, 6, 7498. [CrossRef] [PubMed]

14. Sagawa, T.; Ueda, M. Second law of thermodynamics with discrete quantum feedback control. Phys. Rev. Lett. 2008, 100, 080403. [CrossRef] [PubMed] 
15. Cheong, R.; Rhee, A.; Wang, C.J.; Nemenman, I.; Levchenko, A. Information transduction capacity of noisy biochemical signaling networks. Science 2011, 334, 354-358. [CrossRef] [PubMed]

16. Selimkhanov, J.; Taylor, B.; Yao, J.; Pilko, A.; Albeck, J.; Hoffmann, A.; Tsimring, L.; Wollman, R. Systems biology. Accurate information transmission through dynamic biochemical signaling networks. Science 2014, 346, 1370-1373. [CrossRef] [PubMed]

17. Tsuruyama, T. Channel Capacity of Coding System on Tsallis Entropy and q-Statistics. Entropy 2017, 19, 682. [CrossRef]

18. Aharon, K.K.; Curran, P.F. Nonequilibrium Thermodynamics in Biophysics; Harvard University Press: Cambridge, MA, USA, 2013.

19. BrØnsted, J.N. Principles and Problems in Energetics; Interscience Publishers, Inc.: New York, NY, USA, 1955.

20. Sumino, Y.; Nagai, K.H.; Shitaka, Y.; Tanaka, D.; Yoshikawa, K.; Chate, H.; Oiwa, K. Large-scale vortex lattice emerging from collectively moving microtubules. Nature 2012, 483, 448-452. [CrossRef] [PubMed]

21. Teschendorff, A.E.; Enver, T. Single-cell entropy for accurate estimation of differentiation potency from a cell's transcriptome. Nat. Commun. 2017, 8, 15599. [CrossRef] [PubMed]

22. Jin, S.; MacLean, A.L.; Peng, T.; Nie, Q. scEpath: Energy landscape-based inference of transition probabilities and cellular trajectories from single-cell transcriptomic data. Bioinformatics 2018, 1, 1-10. [CrossRef] [PubMed]

23. Sato, M.; Kawana, K.; Adachi, K.; Fujimoto, A.; Yoshida, M.; Nakamura, H.; Nishida, H.; Inoue, T.; Taguchi, A.; Ogishima, J.; et al. Intracellular signaling entropy can be a biomarker for predicting the development of cervical intraepithelial neoplasia. PLoS ONE 2017, 12, e0176353. [CrossRef] [PubMed]

24. Teschendorff, A.E.; Banerji, C.R.; Severini, S.; Kuehn, R.; Sollich, P. Increased signaling entropy in cancer requires the scale-free property of protein interaction networks. Sci. Rep. 2015. [CrossRef] [PubMed]

25. Banerji, C.R.; Severini, S.; Caldas, C.; Teschendorff, A.E. Intra-tumour signalling entropy determines clinical outcome in breast and lung cancer. PLoS Comput. Biol. 2015, 11, e1004115. [CrossRef] [PubMed]

26. Dopazo, J.; Erten, C. Graph-theoretical comparison of normal and tumor networks in identifying BRCA genes. BMC Syst. Biol. 2017, 11, e110. [CrossRef] [PubMed]

(C) 2018 by the author. Licensee MDPI, Basel, Switzerland. This article is an open access article distributed under the terms and conditions of the Creative Commons Attribution (CC BY) license (http:/ / creativecommons.org/licenses/by/4.0/). 